Results 3894 pregnant women were included in the study. 3842 singleton pregnancies were analysed for adverse neonatal outcomes. $23.5 \%$ of pregnant women were identified as having a mental health condition at the time of antenatal booking. $16.4 \%$ of pregnant women had a known history of depression. $33 \%$ of these women took antidepressants during pregnancy. $37.9 \%$ of pregnant women with history of depression are known to be cigarette smokers compared to $18.5 \%$ of those with no known mental health condition. $65 \%$ of babies with low birth weight, born to mothers taking antidepressants, were associated with positive maternal smoking (OR 3.03, CI: 1.16 to $7.97, \mathrm{p}=0.02$ ).

Conclusion Mental health conditions are common in pregnancy. Babies born to mothers with known history of depression are more likely to be premature, have low birth weight and be admitted to the neonatal unit.

\section{G197(P) A SYSTEMATIC REVIEW OF QUALITY IMPROVEMENT INITIATIVES IN LOW AND MIDDLE INCOME COUNTRIES FOR HOSPITALISED SICK AND SMALL NEONATES}

${ }^{1} \mathrm{~N}$ Zaka, ${ }^{2} \mathrm{EC}$ Alexander, 1,3 $\mathrm{L}$ Manikam, ${ }^{2} \mathrm{I}$ Norman, ${ }^{2} \mathrm{M}$ Akhbari, ${ }^{4} S$ Moxon, 5,6PK Ram, ${ }^{7} \mathrm{G}$ Murphy, ${ }^{7} \mathrm{M}$ English, ${ }^{6,8} \mathrm{~S}$ Niermeyer, ${ }^{1} \mathrm{~L}$ Pearson. ${ }^{1}$ UNICEF, New York, USA; ${ }^{2}$ GKT School of Medical Education, King's College London, London, UK; ${ }^{3}$ Population, Policy and Practice, UCL Great Ormond Street Hospital Institute of Child Health, University College London, London, UK; ${ }^{4}$ Department of Infectious Disease Epidemiology, London School of Hygiene and Tropical Medicine, London, UK; ${ }^{5}$ Department of Epidemiology and Environmental Health, University at Buffalo, New York, USA; ${ }^{6}$ Office for Maternal and Child Health and Nutrition, USAID, Washington, USA; ${ }^{7}$ Centre for Tropical Medicine and Global Health, University of Oxford, Oxford, UK; ${ }^{8}$ School of Medicine, University of Colorado, Aurora, USA

\subsection{6/archdischild-2018-rcpch.192}

Aims $98 \%$ of the estimated 2.7 million neonatal deaths in 2015 occurred in low- and middle-income countries (LMICs). Within this group, neonates who are preterm or small for gestational age are at higher risk given the notable incidence of cardiopulmonary and neurodevelopmental disorders, and infectious complications, in this group. Quality Improvement (QI) initiatives can reduce the burden of morbidity and mortality for hospitalised neonates in LMICs. We undertook a systematic review to synthesise evidence from LMICs on QI approaches used, outcome measures employed, and the nature of any barriers or promoters for efficacy.

Methods Database searching included Medline, EMBASE, WHO Global Health Library, Cochrane Library, WHO ICTRP and ClinicalTrials.gov, and we conducted citation searching of identified studies and reviews. Search terms were 'neonates', 'quality improvement', 'hospitalised' and derivatives. Studies were excluded if they took place in high income countries, did not include QI interventions, did not include small/sick hospitalised neonates. Included studies were published between January 2000-April 2017. Quality appraisal was conducted with use of Cochrane Risk of Bias tools for interventions.

Results A total of 28 studies were included, covering 23 LMICs and 57902 participants. The interventions mostly took place at the district and clinic level, and secondly at the level of patient-provider interaction. Educational interventions were most common, and other popular interventions included service organisation and referencing material dissemination. Mortality was the most commonly assessed outcome, and length of admission, sepsis rates and infection rates were also commonly investigated. Barriers included overburdened staff and insufficient equipment; promoters included motivation of key figures and monitoring.

Conclusion To our knowledge this is the first systematic review to examine QI initiatives in this specific population. The majority of QI initiatives for hospitalised small and sick neonates are aimed at the district and clinic level. Outcomes tended to focus on the delivery of safe and effective care, but rarely focused on people-centred, timely or equitable care. Programme planners should aim for coordinated, larger-scale interventions, sustainable information systems, and to be mindful of barriers.

\section{G198(P) EXPRESS YOURSELF: A QUALITY IMPROVEMENT PROJECT AIMED AT EARLY BREAST MILK EXPRESSION}

CL Granger, G Bowker, A Powls. Neonatal Intensive Care Unit, Princess Royal Maternity Hospital, Glasgow, UK

\subsection{6/archdischild-2018-rcpch.193}

Aim Breast milk reduces rates of necrotising enterocolitis (NEC) which causes significant mortality and morbidity in low birth weight babies. In addition it is closely associated with improved cognitive scores in childhood. Early breast milk allows quicker achievement of full enteral feeds. An internal audit 2015-2016 identified that $61 \%$ of babies $<31$ weeks gestation were commenced on mother's own milk (MOM). Median day of starting MOM was Day 3 and median day of achievement of full enteral feeds was Day 9. We therefore aimed to reduce time taken to establish breast milk expression to postpartum mums of infants $<31$ weeks gestation to under six hours postnatally.

Methods A core team was established to identify barriers to early breast milk expression within the first hour of life. Short surveys were carried out on labour ward and education sessions were arranged on both labour ward and on the neonatal unit to promote the benefits of breast milk to preterm babies. Early expressing packs were put together and given to mums either antenatally or postnatally together with counselling from a senior member of the neonatal team including the benefits of early expressing. (P)s were designed and put up throughout the antenatal ward, neonatal unit and labour ward. Regular feedback to staff involved was facilitated through infant feeding meetings.

Data/Results Following initiation of our QI intervention in November 2016, initial data set audit shows that $73 \%$ of women are being shown how to express within the first 6 hours postnatally, and $94 \%$ of women when questioned are being shown how to express overall. Since introduction, 36 babies have been born $<31$ weeks gestation. $78 \%$ of these were commenced on MOM, median day of starting MOM was Day 1.8 and median day of achievement of full enteral feeds was Day 7.1.

Conclusion With commitment to ongoing education and intervention early breast milk expression is achievable. We have managed to achieve expressing within six hours and with ongoing cycles we are aiming to achieve this within one hour. Next steps include antenatal leaflets for parents and increased scope of education sessions. 